\title{
Response: Cut-off Values and Clinical Utility of Surrogate Markers for Insulin Resistance and Beta-Cell Function to Identify Metabolic Syndrome and Its Components among Southern Indian Adults (J Obes Metab Syndr 2020;29:281-91)
}

\author{
Chiranjeevi Kumar Endukuru', Girwar Singh Gaur ${ }^{1 *}$, Dhanalakshmi Yerrabelli', \\ Jayaprakash Sahoo ${ }^{2}$, Balasubramaniyan Vairappan ${ }^{3}$ \\ Departments of ${ }^{1}$ Physiology, ${ }^{2}$ Endocrinology, and ${ }^{3}$ Biochemistry, Jawaharlal Institute of Postgraduate Medical \\ Education and Research, Puducherry, India
}

\author{
Received December 9, 2021 \\ Reviewed December 21, 2021 \\ Accepted December 22, 2021 \\ *Corresponding author
}

Girwar Singh Gaur

https://orcid.org/0000-0002-6593-5187

Department of Physiology, Jawaharlal Institute of Postgraduate Medical Education and Research,

Puducherry 605006, India Tel: +91-9994470395

Fax: +91-0413-2272066

E-mail: drgsgaur@yahoo.com
Insulin resistance (IR) is a defining feature of obesity, type 2 diabetes mellitus, and cardiovascular diseases and a major contributing factor in metabolic syndrome (MetS) ${ }^{1,2}$ Although researchers have made tremendous progress in understanding IR, certain elements remain unclear, especially diagnosis. ${ }^{3}$ Various types of tests are available for quantitative estimation of IR, ranging from complicated, invasive, time-consuming procedures to simple blood tests using fasting samples. ${ }^{4}$ The hyperinsulinemic-euglycemic glucose clamp is the gold standard method for determining IR but is impractical as it is both labor- and time-intensive. ${ }^{5}$ This issue has led to development of relatively simple markers for IR. In this study, we explored a variety of surrogate indices which have been currently available for estimating IR, insulin secretion, insulin sensitivity, and beta-cell function in a simplified and improved manner. We also investigated their clinical utility in and cutoff values for identifying MetS and its components. A deeper knowledge of these markers will help us better understand and manage this condition.
We conducted this cross-sectional study as a preliminary approach and recruited 150 participants (75 MetS patients and 75 healthy controls) based on the inclusion and exclusion criteria. While recruiting, we performed an interim analysis with 30 people in each group to obtain the mean age and male-to-female ratio. Based on our interim analysis results, we recruited the remaining participants in the case and control groups after matching them for both age and sex. We performed statistical analysis accordingly after assessing the normality of the data. Using validated formulas, we computed 19 IR and insulin secretion indices derived from fasting glucose, insulin, lipid profiles, and adiponectin. ${ }^{3,4}$ Through a receiver operating characteristic (ROC) analysis, markers with the area under the curve (AUC) values between 0.8 and 1.0 were deemed the most important predictors for detecting MetS. Among the surrogate markers of IR and insulin secretion, homeostatic model assessment of IR (HOMA-IR), HOMA-adiponectin (HOMA-AD), triglyceride-glucose (TyG) index, HOMA-1\%S (insulin sensitivity), 
quantitative insulin sensitivity check index (QUICKI), McAuley index, single-point insulin sensitivity estimator (SPISE), and HOMA-2\%B (beta-cell function) showed better accuracy in identifying people with MetS. ${ }^{6}$

We used MedCalc 11.4.2.0 (Ostend, Belgium) software to compare the AUCs of the studied indices. ' Compared with HOMA1IR, non-significant differences were found with the HOMA-AD and TyG indices. Thus, the HOMA1-IR performed well in screening for MetS. The HOMA-AD and TyG index performed similarly to the HOMA1-IR in identifying MetS ( $P>0.05$ vs. HOMA1-IR). Among the insulin sensitivity and beta-cell function indices, the QUICKI, McAuley index, and SPISE showed non-significant differences compared to the HOMA-1\%S. Thus, the HOMA- $1 \%$ S performed well in screening for MetS. The QUICKI, McAuley index, and SPISE performed similarly to the HOMA- $1 \% \mathrm{~S}$ in identifying MetS $(P>0.05$ vs. HOMA-1\%S).

We also emphasized the clinical efficacy of surrogate markers of IR in diagnosing MetS by employing specific cutoff values. Our study found that the optimal cutoff points for these indices were close to the established international cutoffs and did not converge across populations. We therefore presumed that ethnic, racial, and lifestyle variances; data collection methods; diagnostic approaches; and measurement techniques were the primary reasons for the differences in cutoff values across studies. To be more precise, several pathophysiological factors affecting beta-cell function (such as chronic hyperglycemia, oxidative stress, inflammation, and bodyfat distribution) induce changes in the regulation of gene expression that converge on impaired insulin secretion and beta-cell dysfunction. ${ }^{8}$

We evaluated the AUC and optimal cutoff points for the surrogate markers of IR, insulin secretion, insulin sensitivity, and betacell function, in our ROC analysis to identify MetS cases stratified by sex. Our results contained higher cutoff values in women for markers of IR and insulin secretion and lower cutoff values for markers of insulin sensitivity and beta-cell function than in men. Thus, IR was defined as higher levels of markers of IR and insulin secretion and lower levels of markers of insulin sensitivity and betacell function in women than in men. Adiposity, weight status, body composition, and multiple metabolic abnormalities could explain disagreements in the findings between men and women. Among men, the HOMA1-IR (AUC, 0.812) and fasting insulin resistance (FIRI; AUC, 0.812) indices exhibited a stronger predictive ability for MetS than other IR indices. Similarly, the HOMA-1\%S (AUC, 0.812), QUICKI (AUC, 0.806), Bennet (AUC, 0.821), and fasting insulin sensitivity index (FISI; AUC, 0.812) indices displayed the best predictive ability for MetS for insulin sensitivity markers, while the HOMA-1\%B (AUC, 0.801) and HOMA-2\%B (AUC, 0.800) indices were better able to predict MetS using beta-cell function markers. Among women, HOMA1-IR (AUC, 0.902), HOMA2-IR (AUC, 0.836), HOMA-AD (AUC, 0.948), HOMA-triglycerides (HOMA-TG; AUC, 0.811), TyG index (AUC, 0.898), and FIRI (AUC, 0.902) indices all had a better predictive ability for MetS. Similarly, HOMA-1\%S (AUC, 0.902), HOMA-2\%S (AUC, 0.819), QUICKI (AUC, 0.903), Bennet (AUC, 0.924), and FISI (AUC, 0.902) indices best predicted MetS using insulin sensitivity markers, while the HOMA-1\%B (AUC, 0.877) and HOMA-2\%B (AUC, 0.901 ) indices demonstrated the best ability to predict $\mathrm{MeS}$ with beta-cell function markers.

The HOMA-AD was the strongest predictor of MetS in women among the IR markers and secretion monitors with a cutoff value that varied between $\geq 4.03$ in men and $\geq 4.90$ in women. The HOMA-IR and FIRI indices showed the most substantial predictive ability for MetS in men with varied cutoff values (HOMA-IR $\geq 3.29$ in men and $\geq 3.53$ in women, FIRI $\geq 2.96$ in men and $\geq 3.18$ in women). The Bennet index was the strongest predictor of MetS among the insulin sensitivity and beta-cell function markers regardless of sex, with a cutoff value that varied as $\leq 1.16$ in men and $\leq 1.20$ in women. The Bennet, FISI, and HOMA-1\%B indices in men and the HOMA2-IR, HOMA-TG, FIRI, HOMA$2 \% \mathrm{~S}$, Bennet, FISI, and HOMA-1\%B indices in women displayed better predictive ability for MetS when stratified by sex as an addon to our previous findings. ${ }^{6}$ Both women and men exhibited the lowest AUC values for FIGR and glucose to insulin (GI) ratio, respectively. Therefore, the cutoff points for FIGR and GI ratio in both men and women did not meet the AUC requirements for detecting MetS.

Recent studies have reported similar findings. ${ }^{910}$ A Spanish study calculated higher cutoff points for HOMA-IR in women than in men ( $\geq 2.07$ vs. $\geq 1.85$, respectively). ${ }^{9}$ Another study reported optimal cutoff points of $\geq 2.0$ and $\geq 2.5$ for the HOMA-IR and 
$\leq 0.343$ and $\leq 0.331$ for the QUICKI to diagnose MetS in men and women, respectively. ${ }^{10}$ Consequently, there appears to be a sex difference in the AUCs and cutoff values for all surrogate markers of IR and insulin secretion. Hence, a sex-specific reference value is appropriate in clinical practice. The present study deserves further validation in large, multi-centric population-based studies that include more IR markers involving oral glucose tolerance testing with extended follow-up periods and participants from different ethnic backgrounds.

\section{CONFLICTS OF INTEREST}

The authors declare no conflict of interest.

\section{ACKNOWLEDGMENTS}

We sincerely acknowledge the Jawaharlal Institute of Postgraduate Medical Education and Research (JIPMER) for providing financial help in the form of an intramural $\mathrm{PhD}$ research grant (JIP/ Res/Intramural/Phs-1/2018-19/98). We are also thankful to all the participants who took part in this study.

\section{AUTHOR CONTRIBUTIONS}

Study concept and design: all authors; acquisition of data: CKE; analysis and interpretation of data: GSG, CKE, and DY; drafting of the manuscript: GSG, CKE, and DY; critical revision of the manuscript: all authors; statistical analysis: CKE and DY; obtaining funding: CKE; administrative, technical, or material support: GSG; and Study supervision: GSG.

\section{REFERENCES}

1. Khan SH, Khan AN, Chaudhry N, Anwar R, Fazal N, Tariq M.
Comparison of various steady state surrogate insulin resistance indices in diagnosing metabolic syndrome. Diabetol Metab Syndr 2019;11:44.

2. Chopra AK. Metabolic syndrome or insulin resistance: evolution, controversies and association with cardiovascular disease risk. Indian J Clin Cardiol 2020;1:77-85.

3. Singh B, Saxena A. Surrogate markers of insulin resistance: a review. World J Diabetes 2010;1:36-47.

4. Park SE, Park CY, Sweeney G. Biomarkers of insulin sensitivity and insulin resistance: past, present and future. Crit Rev Clin Lab Sci 2015;52:180-90.

5. DeFronzo RA, Tobin JD, Andres R. Glucose clamp technique: a method for quantifying insulin secretion and resistance. Am J Physiol 1979;237:E214-23.

6. Endukuru CK, Gaur GS, Yerrabelli D, Sahoo J, Vairappan B. Cut-off values and clinical utility of surrogate markers for insulin resistance and beta-cell function to identify metabolic syndrome and its components among southern Indian adults. J Obes Metab Syndr 2020;29:281-91.

7. Lee JG, Lee S, Kim YJ, Jin HK, Cho BM, Kim YJ, et al. Multiple biomarkers and their relative contributions to identifying metabolic syndrome. Clin Chim Acta 2009;408:50-5.

8. Wallace TM, Levy JC, Matthews DR. Use and abuse of HOMA modeling. Diabetes Care 2004;27:1487-95.

9. Gayoso-Diz P, Otero-González A, Rodriguez-Alvarez MX, Gude F, García F, De Francisco A, et al. Insulin resistance (HOMA-IR) cut-off values and the metabolic syndrome in a general adult population: effect of gender and age: EPIRCE cross-sectional study. BMC Endocr Disord 2013;13:47.

10. Motamed N, Miresmail SJ, Rabiee B, Keyvani H, Farahani B, Maadi M, et al. Optimal cutoff points for HOMA-IR and QUICKI in the diagnosis of metabolic syndrome and non-alcoholic fatty liver disease: a population based study. J Diabetes Complications 2016;30:269-74. 